\title{
Mobilité et appropriation des langues.
}

Une réponse à Deborah Meunier

Jérémi Sauvage

\section{(2) OpenEdition}

Journals

Édition électronique

URL : http://journals.openedition.org/rdlc/7032

DOI : $10.4000 /$ rdlc. 7032

ISSN : 1958-5772

Éditeur

ACEDLE

\section{Référence électronique}

Jérémi Sauvage, " Mobilité et appropriation des langues. ", Recherches en didactique des langues et des cultures [En ligne], 16-2 | 2019, mis en ligne le 09 septembre 2019, consulté le 10 décembre 2020

URL : http://journals.openedition.org/rdlc/7032 ; DOI : https://doi.org/10.4000/rdlc.7032

\section{(c) $(1) \Theta \Theta$}

Recherches en didactique des langues et des cultures is licensed under a Creative Commons AttributionNonCommercial-NoDerivatives 4.0 International License 
Jérémi Sauvage

Mobilité et appropriation des langues.

Une réponse à Deborah Meunier

UMR CNRS Praxiling

Université Paul-Valéry Montpellier 3

Jérémi Sauvage est Maître de conférences habilité à diriger des recherches en Sciences du langage à l'université Paul-Valéry - Montpellier 3, membre du laboratoire Praxiling UMR 5267 CNRS. Ses domaines de recherche sont l'acquisition et la didactique des langues, plus particulièrement en phonétique / phonologie.

jeremi.sauvage@univ-montp3.fr

\title{
Résumé
}

Le présent article aura notamment comme objectif de se focaliser sur l'appropriation des langues dans le cadre d'une mobilité, plus particulièrement lors de l'accueil d'étudiants en mobilité encadrée (type Erasmus) dans une université francophone. L'une des problématiques à laquelle nous nous retrouvons régulièrement confrontée est celle de l'accompagnement linguistique en Français langue étrangère, c'est-à-dire en Français sur Objectif Universitaire. Nous proposons donc dans ce papier de revenir sur les enjeux et les processus à l'œuvre dans ces rapports complexes entre apprenant-locuteur, appropriation d'une langue étrangère et mobilité dynamique.

\begin{abstract}
The aim of this paper will be to focus on the appropriation of languages in the context of mobility, especially when hosting students in supervised mobility (Erasmus programs) in a French-speaking university. One of the issues we regularly face is that of language support in French as a foreign language, which is to say in French with specific purposes. We therefore propose in this paper to return to the issues and processes at work in these complex relationships between the learner-speaker, the appropriation of a foreign language and dynamic mobility.
\end{abstract}

Mots-clés : Mobilité ; enseignement-apprentissage du français langue seconde; processus psycho-sociaux; dynamisme et statisme.

Keywords : Mobility; teaching-learning of French as a second language; psycho-social processes; dynamism and statism.

Le présent article aura notamment comme objectif de se focaliser sur l'appropriation des langues dans le cadre d'une mobilité, plus particulièrement lors de l'accueil d'étudiants en mobilité encadrée (type Erasmus) dans une université francophone. L'une des problématiques à laquelle nous nous retrouvons régulièrement confrontés est celle de l'accompagnement linguistique en Français langue étrangère, c'est-à-dire en Français sur objectifs universitaires. Nous proposons donc dans ce papier de revenir sur les enjeux et les processus à l'œuvre dans 
ces rapports complexes entre apprenant-locuteur, appropriation d'une langue étrangère et mobilité dynamique.

\section{Introduction : à partir de Deborah Meunier}

Deborah Meunier s'interroge depuis plusieurs années $(2011 ; 2013$; 2018) sur cette problématique de la mobilité des étudiants, en particulier dans le cadre des dispositifs Erasmus et Erasmus +. Dans cette continuité de réflexion, nous pensons qu'il est pertinent de réfléchir à ce qu'on appelle «mobilités » et «postures mobilitaires » (Huver \& Castellotti, 2012). Ainsi, nous retiendrons deux aspects qui nous semblent primordiaux : comment la Didactique des langues et des cultures (désormais DDLC) s'articule-t-elle avec l'apprentissage des langues ? En d'autres termes, de quoi parle-t-on précisément lorsqu'on parle d'enseignement / apprentissage ? D'autre part, qu'en est-il des dispositifs d'accueil, c'est-à-dire des lieux pédagogiques accueillant ces étudiants en mobilité ? On peut alors s'interroger comme Deborah Meunier sur ce qu'il en est des dynamiques inclusives / ségrégatives liées aux statuts des langues (Bourdieu et le « Marché des langues ») en fonction des types de mobilités dans lesquelles elles s'inscrivent. Ceci nous invite alors à réfléchir aux liens existants entre mobilité spatiale et mobilité linguistique, tout ceci en lien avec l'appropriation des langues.

Castellotti (2017) fait la distinction entre apprentissage et appropriation, c'est-à-dire en se positionnant du point de vue de l'apprenant. Il nous semble que l'apprentissage en tant que tel revêt un caractère académique tandis que l'appropriation se traduit plus sur un plan individuel,celui de la construction de la personne sociale (Bronckart, 1997). Voilà pourquoi, il nous semble essentiel de réfléchir aux processus d'appropriation et aux dispositifs en DDLC qui sont mis à contribution en classe. Ainsi, Deborah Meunier défend l'idée que la posture et cette dimension processuelle sont deux aspects à prendre en compte pour mieux comprendre ce qui se passe du point de vue de l'apprenant-étudiant en mobilité dans son appropriation de la langue française. Cette posture peut alors être déclinée sur plusieurs niveaux : posture normative (représentations sur la langue), pragmatique (représentations sur les usages) et pluri-réflexive (ce qui renvoie au CELV - Centre européen pour les Langues vivantes du Conseil de l'Europe - et aux approches complexes dont nous parlerons infra).

\section{Appropriation et acquisition}

L'expression "acquisition du langage » est éculée depuis longtemps (Delamotte-Legrand, 1999) et pourtant on continue de l'utiliser (Sauvage, 2015). Tout d'abord parce que le terme acquisition en français n'a pas exactement le même sens que son cousin anglo-saxon acquisition. En français on peut «faire l'acquisition » de quelque chose, d'un objet, tandis qu'en anglais, l'acquisition renvoie plus à une possession, c'est-à-dire plus qu'à un acte d'appropriation mais plutôt à l'action de s'approprier. Cette nuance est extrêmement importante dans la mesure où l'action de s'approprier renvoie à une action, à un agir (Bronckart, 1997 ; 2012) et que l'apprenant concerné se trouve de facto dans une situation sociale dans laquelle il est explicitement actif, ce dont ne témoigne pas l'«acquisition du langage » à la française, qui a pu renvoyer par le passé à une posture passive, en particulier dans les années 1960-70 marquées par l'empreinte chomskyenne et/ou psychanalytique de l'époque et son idéologie innéiste. L'appropriation renvoie donc à l'acte de s'approprier et donc à une dynamique, à une activité humaine et sociale (Leontiev, 1975) témoignant de l'effort psycho-social nécessaire et indispensable de la part de l'apprenti (que celui-ci soit enfant ou adulte, en L1 ou dans une autre langue).

Ensuite, parler de «langage » dans ce processus qui nous interroge relève selon nous d'un abus de... langage justement. Une nouvelle fois, la polysémie de « language » en anglais, qui peut renvoyer soit à la faculté de langage soit à la langue, est venue perturber réflexions et 
usages dans la communauté francophone s'intéressant à cette dimension. Lorsque l'on parle de " second language acquisition » aux USA, on y trouve exactement les mêmes réflexions que celles développées en France en « acquisition des langues secondes » (Véronique, 2005 ; 2017 ; Perdue, 2002) ou en didactique du FLE/ FLS ou en didactique de l'Anglais langue étrangère ou seconde. Nous pensons qu'il s'agit bien ici du même terrain et des mêmes objets qui nous préoccupent, à savoir l'« acquisition des LANGUES ». Même si le linguiste peut d'intéresser à la faculté de langage en trouvant un dénominateur commun à toutes les langues, par exemple le rapport signifiant/signifié à l'origine de la construction du signe linguistique (Whitney, 1875 ; Saussure, 1916), il apparaît aujourd'hui, au regard de l'histoire des idées, que l'étude de la faculté de langage est plus un objet de recherche de la philosophie (depuis le Cratyle de Platon jusqu'à Searle 1972 ou Vandervaeken 1984) que de la linguistique proprement dite.

Ainsi, nous préférons parler d'appropriation des langues plutôt que d'acquisition du langage afin de préciser notre pensée et l'ancrage de notre réflexion. On n'enseigne/n'apprend pas le langage mais bien des langues.

\section{Personne sociale, langue et inter}

Un autre point qui nous semble important est de ne pas oublier la situation réelle dans laquelle s'inscrit toute situation linguistique. Si l'on considère qu'un locuteur, quel qu'il soit, est une personne sociale, c'est-à-dire qu'elle répond aux attendus sociaux, psychologiques et culturels nécessaires à une interaction sociale, alors il devient indispensable de considérer cette situation comme un triptyque : le locuteur-apprenant (comme personne sociale) se situe d'un « inter- » (culturel, linguistique, psychique, social... bref, communicatif) grâce à un medium que peut être une langue (Bronckart, 1999). Pour paraphraser Saussure et ses descendants sociolinguistes (Gumperz, 1989), sans communauté, point de linguistique. L'intérêt (la raison d'être ?) d'une langue est de permettre la constitution d'une communauté sociale en lui permettant de communiquer aisément.

Sur un plan didactique, il convient alors de ne pas ignorer cette dimension sociale et interactionniste des usages linguistiques. La recherche a depuis longtemps montré à la fois l'importance du point de vue des apprentissages mais également de celui du fonctionnement (Sauvage, 2015 pour une synthèse).

Concrètement, l'étudiant en mobilité qui, par exemple, vient étudier à l'université Paul-Valéry - Montpellier 3 en bénéficiant du dispositif Erasmus, se retrouvera confronté à des réalités sociales, culturelles (notamment sur le plan éducatif de l'Université française), etc. Ainsi la question de l'accueil de ces étudiants mérite-t-elle d'être posée (Sauvage, Dufour \& Auger, 2017). Tout cela signifie que pour s'approprier le français comme langue d'enseignement universitaire, il ne suffit certainement pas de s'entrainer à faire des exercices de grammaire par dizaines ou d'écouter tous les jours les médias francophones. Nous pensons qu'il existe des spécificités à cette situation et que l'étudiant étranger inscrit dans un cursus francophone nécessite des attentions particulières. Ce sont ces attentions qui nous interpellent et que nous souhaitons mettre en évidence afin d'améliorer l'accompagnement desdits étudiants tout au long de leur cursus en France.

En conséquence, notre inscription dite «interactionniste» pour étudier les processus à l'œuvre dans l'apprentissage des langues est justifiée par les rapports sociaux eux-mêmes qui sont engendrés dans les activités en classe mais également par les objectifs visés : savoir évoluer, notamment sur le plan linguistique, dans un espace social donné. Nous revendiquons donc une approche sociale de l'enseignement-apprentissage des langues, ce qui, au passage, nous conduit à nous interroger sur l'existence d'une "socio-didactique » (Rispail, 2018) ou d'une «didactique cognitive» (Billières \& Spanghero-Gaillard, 2007), ne voyant pas comment une "réflexion didactique» digne de ce nom pourrait être ni sociale, et/ou ni 
cognitive. Il s'agit bien de revendiquer une approche épistémologique pour concevoir une didactique des langues plutôt que de réduire cette didactique à un tropisme. Cependant, il apparaît, en particulier en formation de formateurs, qu'il existe bien une ambiguité dans l'esprit des étudiants à ce propos, certains se revendiquant d'une nouvelle didactique qui serait sociale, alors qu'il ne peut en être autrement.

Enfin, plutôt que de continuer à parler d' « interlangue », terme essentiel dans les recherches sur le bilinguisme (Selinker, 1972; Han \& Tarone, 2014)) et en didactique des langues étrangères/secondes (Arditty, 2005), nous préférons parler d'espace interlinguistique (Sauvage, 2014; Sauvage \& Guiraud, 2015). Cet espace peut s'apparenter à une distance virtuelle entre les langues, n'ayant rien à voir avec le semilinguisme de Skutnabb-Kangas \& Toukomaa (1976) et qui a fait tant de tort au bilinguisme et à l'apprentissage précoce des langues (Corblin \& Sauvage éds, 2010). Cette distance est conditionnée avec la dimension temporelle : plus l'espace est grand, plus le temps nécessaire à sa réduction est long. Ainsi, le mandarin n'est pas une langue plus difficile à apprendre pour le francophone mais elle nécessitera plus de temps du fait de l'importance de cet espace interlinguistique. On n'attribuera donc aucun jugement de valeur (ce qui serait un comble pour un linguiste) mais plutôt un compte-rendu imagé d'une situation complexe car en évolution, donc en instabilité permanente.

\section{Statisme et dynamisme : (im)mobilité et (im)mobilisme}

Il nous semble indispensable d'interroger ces différentes notions qui sont parentes d'un point de vue sémantique et lourde de connotation pour certaines d'entre elles.

Nous avons déjà exposé notre point de vue sur la dichotomie statisme / dynamisme (Sauvage, 2015) à propos des conceptions épistémologiques de différents domaines scientifiques, en particulier en mathématiques, en philosophie, en psychologie et en linguistique. Cette conception épistémologique résulte le plus souvent d'une posture idéologique, c'est-à-dire d'un choix arbitraire : soit je considère le Cosmos en mouvement, soit je le conçois comme figé. Traditionnellement et pour aller à l'essentiel, on peut dire que l'anthropocentrisme prégaliléen implique une conception statique du Monde. Les choses sont ainsi parce qu'elles sont ainsi, ce qui est sécurisant et simplifiant pour décrire et comprendre le Cosmos. Mais en considérant que le figement, l'équilibre d'un système, n'est qu'une courte période entre deux moments de déséquilibre permettant l'évolution de ce système, on arbore une conception à proprement parler dynamique (et systémique en l'occurrence dans cet exemple). En acquisition / didactique des langues et des cultures, cela revient à une conception active de l'apprenant (quel que soit son statut - bébé, enfant, adulte, élève...) qui doit lui-même agir avec les autres dans le cadre d'activités humaines (Leontiev, 1975) comme l'enseignementapprentissage (Bronckart, 1997 ; Sauvage, 2003).

En jouant sur les mots, il devient possible de s'interroger sur la mobilité d'une personne (étudiante ou non) et/ou sur l'absence de cette mobilité (Meunier, 2012 ; Sauvage, Dufour \& Auger, 2017), c'est-à-dire à son immobilité, conduisant ainsi à une posture statique. Or, il nous semble que la non-mobilité d'un étudiant ne prédispose pas de son immobilisme. La question est alors de savoir si la mobilité est forcément un gage de dynamisme. Un étudiant en mobilité qui ne ferait pas les actions (les agirs, Bronckart, 2012) pourrait se trouver dans une forme d'immobilisme tout en étant en mobilité : en refusant par exemple de s'investir dans l'apprentissage et ses méthodes particulières dans l'université d'accueil. Au-delà de la frontière géographique, la frontière interculturelle joue son rôle en tous sens. Si l'étudiant se doit d'être actif de son point de vue, il convient également de lui proposer des activités idoines pour lui permettre de réussir. C'est notamment sur ce point que nous nous étions déjà interrogés, à propos de l'accompagnement en FLE pour les étudiants en mobilité encadrée (Sauvage, Dufour \& Auger, 2017). 
Une vision statique ou dynamique renvoie donc à des visions différentes du monde et, pour le chercheur, à des épistémès différentes. Ainsi, ce qui peut caractériser un "déséquilibre » dans l'apprentissage d'une langue, comme les variations articulatoires par exemple (Sauvage, 2002 ; 2015) témoignent d'une évolution systémique. La mobilité, comme principe de déséquilibre devient alors par essence le facteur d'une dynamique d'appropriation linguistique possible.

\section{DDLC et étiquettes pour caractériser la langue française}

Un autre point qui nous semble important en réponse à Deborah Meunier est la question des " étiquettes » pour caractériser l'enseignement-apprentissage du français à des apprenants allophones. A l'heure où l'on envisage la disparition du FLI (Français langue d'intégration), ces étiquettes (Robert, 2015) de FLE (Français langue étrangère), FLS (Français langue seconde), FLSco (Français langue de scolarisation), FOS (français sur objectifs spécifiques), FOU (Français sur objectifs universitaires), FLI (Français langue d'intégration), FLM (Français langue maternelle), FL3 (Français langue 3) etc... sont devenues avec le temps sensiblement contreproductives. Le FLI, par exemple prétend se distinguer du FLE car réservé à des adultes migrants ayant un projet d'intégration. Mais le FLE s'utilise comme étiquette quelle que soit la finalité de son apprentissage (y compris l'intégration). L'origine de cette expression, qui n'est donc pas un réel concept scientifique, renvoie explicitement à une vision politique (idéologique) de la fin des années 2000 et on gardera à l'esprit que la communauté scientifique par la voix de ses associations les plus importantes (ASDIFLE, Acedle, Adeb...) dans son ensemble à quelques rares exceptions près (Adami, 2012) s'est opposée à la création de cette nouvelle étiquette lourdement connotée sur le plan culturel et surtout politique. Toujours est-il qu'à l'heure où l'on parle plus volontiers d'inclusion que d'intégration, l'étiquette FLI n'a plus lieu d'être.

Concernant les mobilités étudiantes, il est également possible de parler de Français sur objectifs universitaires ou de cours de Français sur objectifs spécifiques. Le danger avec ces étiquettes est de sous-entendre qu'il existe plusieurs langues françaises à enseigner alors même que tous les centres universitaires de langue française n'ont dans leur dénomination que le terme de FLE. Le didacticien aura à cœur de proposer des hyponymes dans le but de spécifier ses analyses au niveau de son enseignement et de sa recherche. Mais en aucun cas ces étiquettes n'ont à exister à un autre niveau. Enseigner le français comme langue étrangère (FLE) c'est notamment savoir s'adapter à un contexte d'enseignement et/ou à un public spécifique. Cela recouvre de facto l'ensemble des spécificités d'enseignement nécessitant des comportements et des réflexions didactiques. Il nous semble qu'en didactique du français, il n'y pas d'autres dichotomies que la didactique du Français langue maternelle vs la didactique du Fançais langue non-maternelle (Sauvage, 2014). Et encore, il ne faut pas oublier le continuum existant entre ces deux types de didactique, certainement beaucoup plus proches que leur séparation institutionnelle (entre un master MEEF permettant de devenir enseignant dans l'Education nationale et un master FLE permettant d'enseigner le FLE en dehors de l'Education nationale par exemple).

En conséquence, lorsque l'on envisage un «accompagnement en langue française » pour les étudiants en mobilité encadrée (Sauvage, Dufour \& Auger, 2017), il s'agit bien de FLE. Bien entendu, la durée d'un tel programme ne pouvant que difficilement dépasser 40h par semestre, le choix des objectifs est prioritairement tourné vers des compétences générales propres à la langue française en milieu universitaire. Mais il s'agira bien de cours de FLE à part entière.

\section{Moi dans l'espace de l'Autre}

Qui suis-je? 
La place du je (de soi) dans l'apprentissage des langues revêt un statut particulier nécessitant une réflexion à part entière. La construction identitaire en contexte plurilingue et pluriculturel (Demougin \& Sauvage, 2011; Sauvage \& Demougin, 2012) s'avère en effet d'une telle complexité que la construction d'une relation sociale et sociocognitive avec autrui n'est pas aussi évidente que l'on pourrait le penser de prime abord. Si l'on considère que nous avons chacun un espace (social) personnel, l'interaction sociale telle que Vygotski (1934) l'a théorisée revient à considérer une relation entre deux «espaces personnels". En fin de compte, mes interactions avec autrui peuvent être considérées comme des interactions entre moi (mon espace) et l'autre (son espace). Faire bouger mon espace a donc pour conséquence de me faire sortir de ma zone de confort social. Mais finalement, d'un point de vue systémique (Morin, 1994; Sauvage, 2015), la seule question à se poser est celle du déséquilibre et de la rééquilibration. Lorsque je suis dans l'espace de l'autre, je suis en déséquilibre. Il apparaît (d'un point de vue mathématique) une quantité de désordre, d'entropie qui symbolise finalement la dynamique grâce à laquelle un nouvel équilibre sera atteint (Prigogine, 1994). La signification du déséquilibre comme moteur d'évolution (Piaget, 1946 ; Bronckart, 1997 ; Le Moigne, 1977 ; 1990 ; Sauvage 2015) s'inscrit dans une vision dynamique. L'objectif du déséquilibre comme courte période d'inconfort est bien de viser un nouveau plateau de stabilité ?, une nouvelle stabilité, un nouvel équilibre, ce que Piaget appelait en psychologie assimilation, accommodation, adaptation.

La prise de risque de la personne en mobilité, de l'étudiant international en particulier, se traduit donc par le passage par une zone d'inconfort pour mieux évoluer vers une nouvelle stabilité. Tout ceci, nous semble-t-il, met en évidence la nature complexe des enjeux de la mobilité : je suis un autre pour un autre je et l'interactivité entre personnes sociales conduit immanquablement à une dynamique d'évolution (transformation de soi ?). Voilà pourquoi je suis toujours l'autre d'un je(u).

\section{Conclusion}

La mobilité peut donc être considérée comme un vecteur d'appropriation linguistique, dans le sens où les enjeux psycho-sociaux et psycho-affectifs ont un rôle important dans ces processus d'appropriation ainsi que dans leur nature. C'est pourquoi nous avons essayé d'insister dans cet article sur la dimension prépondérante que revêtent la conscience et les représentations des apprenants dans ces processus. Les enjeux à l'œuvre dans l'appropriation linguistique dépassent largement le cadre stricto-sensu de la langue (et peut-être même de sa dimension culturelle). Le chemin du processus participe en effet à cette appropriation et mérite donc, également, d'être considéré comme un objet de recherche et de réflexion. La posture de l'apprenant, face à la mobilité, renvoie donc à une multitude d'aspects, certains pouvant se situer parfois, en apparence, assez loin de ses préoccupations premières. Mais nous pensons que cette recherche d'équilibre, répondant à une déstabilisation permanente, constitue déjà à elle seule une dynamique motrice de l'apprentissage linguistique.

Un dernier point nous paraît essentiel dans cette conception : comment tenir compte de cette mouvance dans la formation des enseignants?

La formation de formateurs, que celle-ci soit initiale (Masters) ou continue, se doit, à notre avis, de prendre en compte cette dimension peu considérée de l'enseignement-apprentissage des langues étrangères. Nous proposons donc d'intégrer dans les curricula des formations cette dimension d'appropriation à la mobilité, sous la forme d'une éducation à la mobilité. On le sait, les étudiants français ne sont pas les plus nombreux en Europe pour bénéficier d'une mobilité encadrée de type Erasmus. Peut-être que la principale raison en est que rien ni personne, de manière académique, les éduque sur les bienfaits et les enjeux de cette mobilité 
(à l'exception d'une simple réunion d'information). Ne pas craindre ce qui n'est pas stable serait alors, assurément, un objectif tout à fait louable à viser dans nos cursus universitaires.

\section{Références bibliographiques}

ADAMI, H. (2012). « La formation linguistique des migrants adultes ». Savoirs, n²9(2). p. 9-44.

ARDITTY, J. (2005). «Approches interactionnistes. Exemples de fondements théoriques et questions de recherche ». In BIGOT, V. \& CICUREL, F. (éds.), Le français dans le monde. Recherches et applications. p.8-19.

BILLIERES, M., \& SPANGHERO-GAILLARD, N. (2007). « La didactique cognitive des langues au service de la méthodologie de l'enseignement-apprentissage du français langue étrangère et seconde ». Entre Lenguas, n¹2. p. 99-114.

BRONCKART, J.P. (1997). Activité langagière, textes et discours : pour un interactionnisme sociodiscursif. Genève : Delachaux \& Niestlé.

BRONCKART, J.P. (1999). "La conscience comme 'analyseur' des épistémologies de Vygotski et Piaget ». In CLOT Y, (Ed.), Avec Vygotski. p.17-43.

BRONCKART, J.-P. (2012). " Le rôle de la maîtrise langagière dans le développement identitaire des personnes ». In SAUVAGE, J. \& DEMOUGIN, F. (Eds). La construction identitaire à l'école. Perspectives linguistiques et plurielles. Paris : L'Harmattan.p. 15-32.

CORBLIN, C. \& SAUVAGE J. (éds). (2010). L'apprentissage et l'enseignement des langues vivantes à l'école. Impacts sur le développement de la langue maternelle. Paris : L'Harmattan.

DEMOUGIN ,F. \& SAUVAGE, J. (2011). «Construction identitaire à l'école. Perspectives didactiques ». Tréma, $\mathrm{n}^{\circ} 33-34$.

GUMPERZ, J.J. (1989). Engager la conversation: introduction à la sociolinguistique interactionnelle. Paris : Editions de Minuit.

HAN, Z. \& TARONE, E. (2014). Interlanguage : forty years later. Amsterdam : Benjamins.

LE MOIGNE, J.-L. (1977). La théorie du système général. Théorie de la modélisation. Paris : PUF.

LE MOIGNE, J.-L. (1990). La modélisation des systèmes complexes. Paris : Dunod.

LEONTIEV, A.N. (1975, 1984). Activité, conscience et personnalité. Paris : Editions du Progrès.

MEUNIER, D. (2011). « Mobilité et apprentissage linguistique : étude du discours métalinguistique d'apprenants Erasmus », Études de Linguistique Appliquée, $\mathrm{n}^{\circ}$ 162., p. 137-151.

MEUNIER,D. (2013). Les représentations linguistiques' des étudiants Erasmus et la vision plurilingue européenne : normes, discours, apprentissages. Thèse de doctorat, Université de Liège et Université Libre de Bruxelles.

MORIN, E. (1994). La complexité humaine. Paris : Flammarion.

MORIN, E. (1999). Relier les connaissances. Paris : Le Seuil.

PERDUE, C., BENAZZO, S. \& GIULIANO, P. (2002). "When finiteness gets marked: the relationship between morpho-syntactic development and use of scopal items in adult language acquisition". Linguistics, $\mathrm{n}^{\circ}$ 40(4). p.849-890.

PRESCOD, P. \& ROBERT, J.-M. (éds.), (2015). «La langue seconde de l'école à l'université. Etat des lieux », Cahiers de Sociolinguistique, $\mathrm{n}^{\circ} 10$. Paris : L’Harmattan.

RISPAIL, M. (2018). Abécédaire autour de la sociodidactique. Saint Etienne : Presses de l'Université de Saint Etienne.

SAUVAGE, J. \& GUIRAUD, F. (2015). «Acquisition d'une récursivité syntaxique en français langue seconde : Exemple du pronom relatif ». In Carnets d'Atelier Sociolinguistiques, ${ }^{\circ}{ }^{\circ}$. $p$. 103-116.

SAUVAGE, J. \& DEMOUGIN, F. (éds.). (2012). «La construction identitaire à l'école. Perspectives linguistiques et pluriculturelles ». Actes du colloque «TITRE DU COLLOQUE » Paris : L'Harmattan.

SAUVAGE, J. (2002). «Phonological development in French : the part of lexical dimension in the variation". In B. LEWANDOWSKA-TOMASZCZYK \& K. TUREWICZ (éds) (2002): Cognitive Linguistics Today, Berlin : Peter Lang, 571-581. 
SAUVAGE, J. (2003). L'acquisition du langage chez l'enfant. Approche dynamique et développementale. Paris : L'Harmattan.

SAUVAGE, J. (2014). "Acquisition et didactique du FLS : processus psychosociaux et interactionnisme socio-discursif ». In Études de Linguistique Appliquée, n¹74.p. 157164.

SAUVAGE, J. (2015). L'Acquisition du langage un système complexe. Louvain-la-Neuve : Academia.

SAUVAGE, J., DUFOUR, S. \& AUGER, N. (2017). «Face à l'hétérogénéité des projets de mobilité ERASMUS: Evaluer les enseignements de FLE de l'université Paul-Valéry Montpellier $3 »$. Le Langage et l'Homme, n`52-1, p. 77-92.

SELINKER, L. (1972). Interlanguage. International Review of Applied Linguistics, 10, 219231.(norme?)

SKUTNABB-KANGAS, T. (1984). Bilinguism or not? Clevedon, Avon: Multilingual Matters.(idem)

SKUTNABB-KANGAS, T. \& TOUKOMAA, P. (1976). Teaching Migrant Children's mother tongue and Learning the Language of the Host Country in the Context of Social-Cultural Situation of the Migrant Family. Tampere: UNESCO report, University of Tampere, Research Report, 15. (norme ?)

VERONIQUE, G.D. (2005). "Les interrelations entre la recherche sur l'acquisition du français langue étrangère et la didactique du français langue étrangère. Quelques pistes de travail ». AILE, n०23. p.9-41.

VERONIQUE, G.D. (2017). « La grammaire en français langue étrangère : questions d'acquisition et d'intervention », Lidil [En ligne], 56 | 2017, mis en ligne le 01 novembre 2017, consulté le 11 février 2019. URL : http://journals.openedition.org/lidil/4734

VYGOTSKI, L. (1934-97). Langage et pensée. Paris : La Dispute. 